Document downloaded from:

http://hdl.handle.net/10251/40408

This paper must be cited as:

Picó Vila, R.; Pérez Arjona, I.; Sánchez Morcillo, VJ.; Staliünas, K. (2013). Evidences of spatial (angular) filtering of sound beams by sonic crystals. Applied Acoustics. 74(7):945948. doi:10.1016/j.apacoust.2013.01.003.

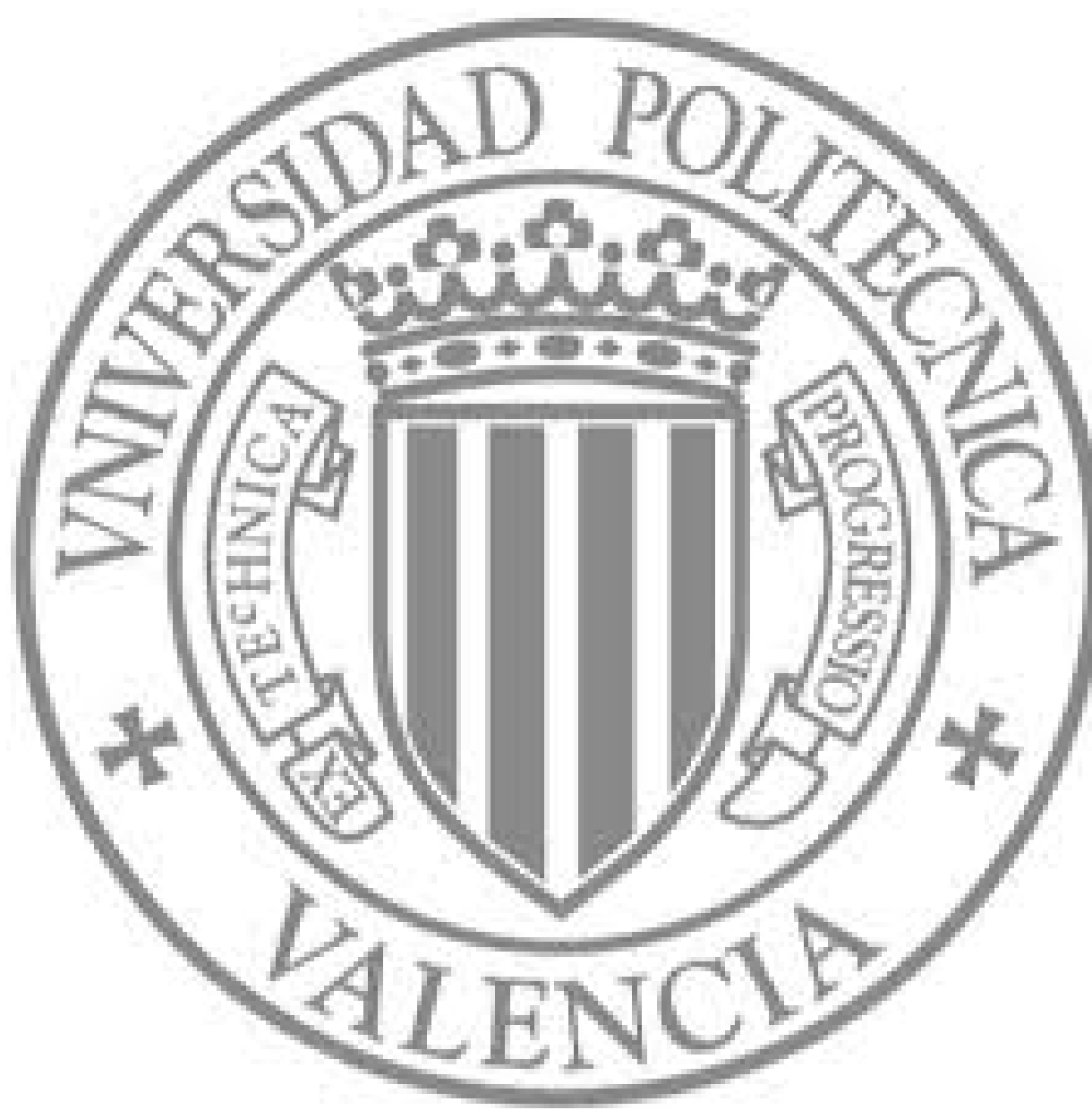

The final publication is available at

http://dx.doi.org/10.1016/j.apacoust.2013.01.003

Copyright Elsevier 


\title{
Evidences of Spatial (Angular) Filtering of Sound Beams by Sonic Crystals
}

\author{
R. Picó ${ }^{1}$, I. Pérez-Arjona ${ }^{1}$, V. J. Sánchez-Morcillo ${ }^{1}$ and K. Staliunas ${ }^{2}$ \\ ${ }^{1}$ Instituto de Investigación para la Gestión Integrada de Zonas Costeras, Universidad \\ Politécnica de Valencia, Paranimf 1, 46730 Grao de Gandia, Spain \\ ${ }^{2}$ ICREA, Departament de Fisica i Enginyeria Nuclear, Universitat Politècnica de \\ Catalunya, Colom, 11, E-08222 Terrassa, Barcelona, Spain
}

\begin{abstract}
We report experimental evidences of spatial (angular) filtering of sound beams propagating through sonic crystals. We show that at specific frequencies of the incident wave the paraxial plane wave components of the beam can be efficiently transmitted through the crystal, whereas the components propagating at large angles are strongly reflected or deflected (filtered out) by the crystal. The modification of the angular field distribution results in formation of sound beams of relatively high spatial quality.
\end{abstract}

\section{1.- Introduction}

It is known that transmission of waves through spatially modulated materials, where the modulation period is of the order of magnitude of the wavelength (photonic crystals in optics, sonic or phononic crystals in acoustics) can be sensible to the direction of propagation. Modulated materials can be transparent for the waves propagating along one direction, but can be strongly reflecting or deflecting for the waves of the same frequency but propagating at other angles. The material can be strongly anisotropic, or in other words, can show angular band-gaps. An idea has been recently proposed in optics, that the angular sensitivity of wave transmission could be utilized for spatial filtering of monochromatic wave beams [1-4]. The proposals are based on the observation that the modulation of the material can be chosen so, that the paraxial plane wave components (those forming small angles to the beam axis) can propagate through the material, but non-paraxial components (those forming larger angles to the beam axis) can be efficiently reflected or deflected. This results in narrowing of the angular distribution (spatial spectrum) of the beam, and leads to low-pass angular filtering. The phenomenon of spatial filtering is analogous to the well known effect of frequency filtering in one-dimensionally modulated materials, which is based on the existence of frequency band-gaps. The wave components with the frequencies within the 
propagation bands can be transmitted through, whereas the frequencies in band-gaps experience strong reflection from such band-gap materials. The frequency bandgaps have been extensively studied in optics, for photonic crystals [5], and more recently in acoustics, for sonic crystals (SC) [6]. We note that one-dimensional modulation (i.e. the layering) is sufficient for the observation of frequency bandgaps, therefore is also sufficient for realization of the frequency filtering.

The spatial filtering is possible only for materials with two- or three-dimensional modulation in space. After the theoretical proposals [1-4] some experimental evidences of spatial filtering have been reported in optics, for photonic crystals [7]. The spatial filtering has been also proposed for matter waves (Bose-Einstein condensates in spatially and temporally modulated potentials) [8]. Recently the spatial filtering has been theoretically predicted in acoustics, for two-dimensional sonic crystals of rhombic symmetry [9]. Here we demonstrate experimentally the spatial filtering phenomenon in acoustics for the first time. Differently from the theoretical predictions in [9] we consider in this work a square geometry of the crystal. Differently from the experimental demonstration in optics [7] the effect in acoustics is very strong: a substantial portion of off-axis radiation can be filtered-out from the beam. In the present letter we show, that the effect of spatial filtering can be efficient enough, and can be utile for cleaning the spatial structure of the beam, i.e. for formation of high spatial quality beams from the low quality sources.

\section{2.- Experimental setup}

The experimental scheme used to demonstrate the spatial filtering is shown in Fig. 1. The SC is a matrix of 9x10 cylindrical (methacrylate) scatterers of radius $r=1 \mathrm{~cm}$, in square lattice with lattice constant $a=4.7 \mathrm{~cm}$, surrounded by air. The Bragg frequency for the given geometry, i.e. the frequency corresponding to the center of the first banggap, is $f_{b}=c / 2 a=3600 \mathrm{~Hz}, c$ being the sound velocity in air. The filling fraction of the crystal $f=\pi(r / a)^{2}=0.14$ was chosen relatively small in order to keep the transmittance high. The experiment was performed inside an anechoic chamber, in order to avoid undesired sound reflections from the room walls. The emitter was a broad band loudspeaker emitting white noise, and the receiver was a microphone mounted on a rotating axis controlled by a robot. The fast Fourier transformation was computed from the signals in time domain recorded by a microphone in order to get the frequency spectra. The frequency resolution is $1 / 48^{\text {th }}$ of the central frequency of the band, and the angular precision was the robot rotation step $\left(\Delta \theta=2.5^{\circ}\right)$. Measurements covered the range from $0^{\circ}$ to $180^{\circ}$ (not all $360^{\circ}$ because of the symmetry of the system). That makes in total the measurement at 73 scattering angles. 

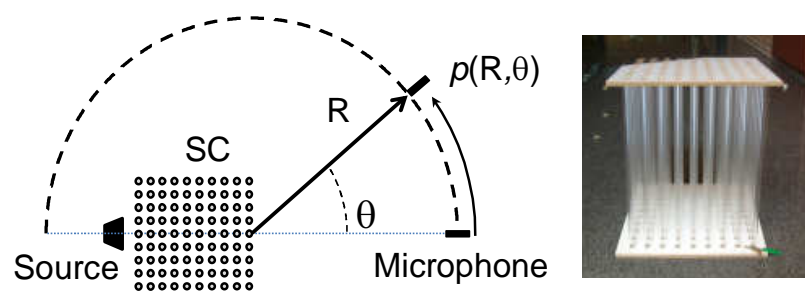

Fig.1. Experimental set-up consisting of the source (loudspeaker), the sonic crystal, and a mobile point-detector (microphone). On the right side a photograph of the sonic crystal is given.

\section{3.- Band structure and Isofrequency contours}

A theoretical analysis was performed for the parameters corresponding to the experimental setup. Angular gaps are most clearly evidenced by calculating and plotting the dispersion diagrams by plane wave expansion method [10], and plotting the isofrequency contours in k space. Fig. 2 shows the band structure in standard ГХМГ representation [2(a)], and the isofrequency contours [2(b) and 2(c)] at two frequencies where the spatial filtering is expected.

(a)

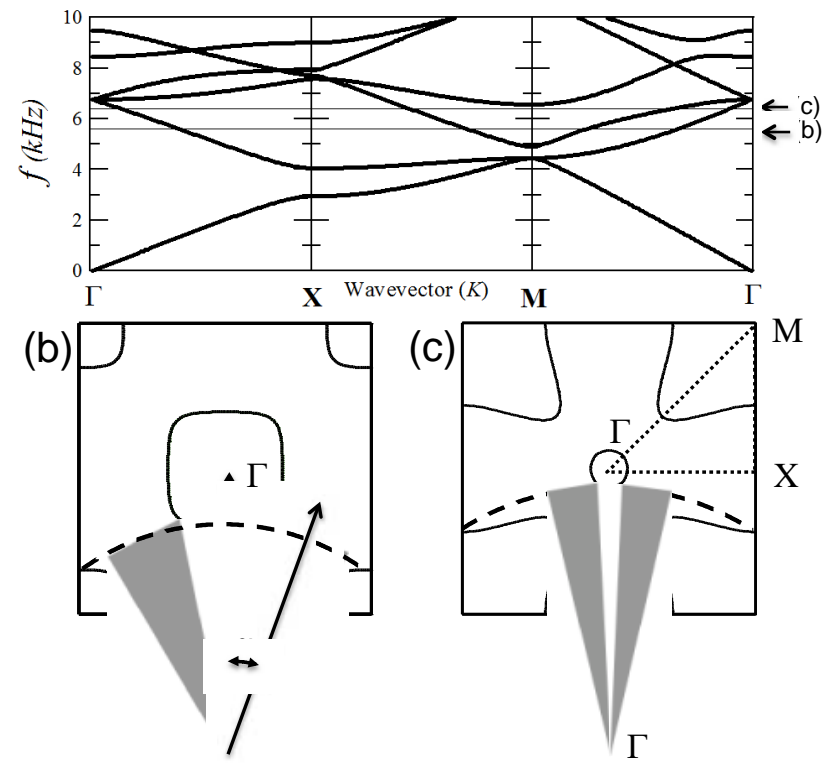

Fig.2. (a) Band-diagram showing the eigenfrequency dependence on the wavevector along the standard ГХМГ path; $(b, c)$ isofrequency contours showing the angular band-gaps between the second and the third Bloch band for frequencies $f_{1}=5.4 \mathrm{kHz}$ and $f_{2}=6.4 \mathrm{kHz}$ for $(b)$, and (c) respectively, as indicated by horizontal continuous and dashed lines in (a). The angular bandgaps in (b) and (c) are indicated by shaded areas. 
The $\omega(k)$ dispersion curves in Fig. 2(a) show the frequency band-gaps. For a low filling factor considered here, only a partial gap (frequency gap for some particular directions) is obtained. Close to the top of the bands (in the case shown in the Fig. 2, close to the top of the second band) the isofrequency contours are strongly distorted with respect to those in homogeneous space (the concentric circles). It is known that the isofrequency contours can show flat segments at particular frequencies, which are at the basis of the self-collimation theoretically predicted [11] and experimentally observed [12] for the SCs. For larger frequencies, the dispersion lines with positive sign of curvature appear around the X-point, responsible for the negative diffraction. However between the isofrequency curve in the second band (around the X-point) and strongly curved isofrequency lines in the third band, the angular band-gaps appear, as indicated by the shadowed areas in Figs. 2(b) and (c): the plane wave components at corresponding angles can not propagate and are reflected back or deflected. The position and width of the angular band-gaps depends on the frequency as Figs. 2(b) and (c) evidence.

The angular band-gaps are at the basis of the spatial filtering and cleaning of beams. For initially broad beams, with relatively narrow angular spectrum, the spatial filtering effect would result in a narrowing of the far field distributions of the beams. For narrow beams, with relatively broad angular spectrum, the filtering could result in depletion of particular angular ranges of the spatial spectra.

The predictions by the plane wave expansion have been checked by numerical simulation of the beam propagation through the SC using the finite element method [13]. The domain of the model is bounded (absorbing boundaries) at a distance corresponding to the measurement positions in the experimental set-up. The acoustic excitation has been introduced in the model as a Gaussian profile for the pressure in the form $p(y)=\exp \left(-y^{2} / \sigma^{2}\right)$, where $\sigma$ is related with the width of the source. In all simulations in this work $\sigma=0.01 \mathrm{~m}$. Due to extremely high contrast of acoustic impedance between material and air, all the scatters have been considered as rigid. A Perfectly Matched Layer (PML) has been used at the external bounds of the domain in order to avoid undesired reflections. The hardware used for the simulations is a Fujitsu Celsius R670-2 with 24 cores, $48 \mathrm{G}$, which resulted in computational time for one frequency excitation less than 5 seconds. The computation time for the complete model is less than 1 minute. 


\section{4.- Numerical results}

The results are summarized in Fig. 3, which shows the angular distributions of the sound (horizontal axis) versus the frequency (vertical axis) in Fig. 3(a), and also the spatial distribution of the sound intensity in the beams at particular frequencies, behind the crystal [Figs. 3(b)-(e)]. For comparison, in order to highlight the filtering effect of the crystal, also the distributions of the field diffracted by a rigid block with the size of the $\mathrm{SC}$, also the freely propagating beam are shown in Figs. 3(f) and (g) respectively.

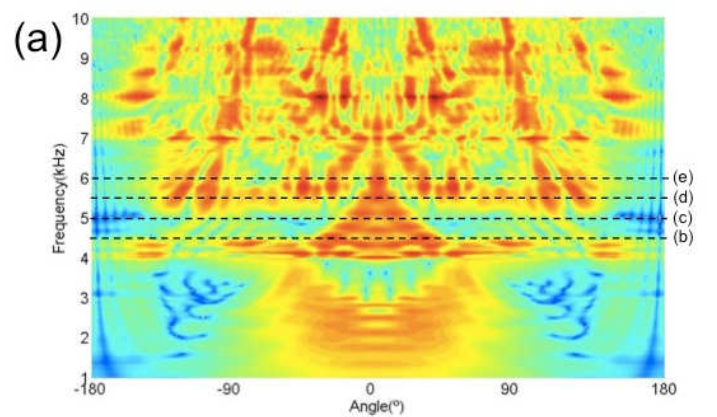

(b)

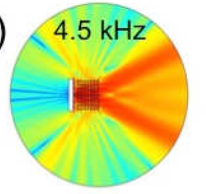

(c)

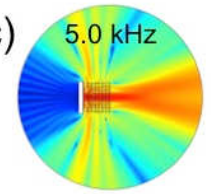

(d)

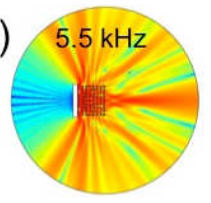

(f)

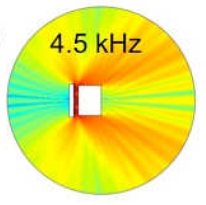

(e)

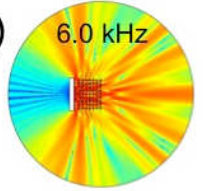

(g)

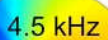

Fig.3. (a) Far field (angular) distributions of the amplitude of the sound beam transmitted through the crystal, as obtained by FEM numerical simulations in terms of the input frequency. Figures (b), (c), (d) and (e) show the spatial sound distribution at the indicated frequencies. The parameters are as in Fig. 2.

The frequency was varied in a broad range, covering several frequency band-gaps and also covering the expected area of spatial filtering (as following from the analysis of the spatial dispersion curves presented above). For low frequencies $f<f_{b}$, well below the first band-gap in the considered $\Gamma X$ direction, the sound field spread strongly. The spatial structure of the crystal is "invisible", i.e. the sound waves propagate in the crystal as in a homogeneous medium with effective parameters (the so-called 
homogenization limit). For frequencies corresponding to the second propagation band the angular transmission spectrum becomes very sensitive to the frequency and the angular band-gaps appear at particular directions (see e.g. the field plot for $f=4.5 \mathrm{kHz}$ ). At the top of the second band, according to the spatial dispersion diagrams, the expected effect of spatial filtering is observed (see field plots for different frequencies, Figs 3(c), (e)). Increasing the frequency the transmission angle decreases, and the beam well behind the SC becomes narrower. For higher frequencies, Bloch modes from the third and higher propagation bands come into play. The spatial filtering is possible only for the beams of relatively narrow angular spectrum. For beams with broad angular spectrum depletion appears in the transmitted angular spectrum, and no "clean" lowpass angular filtering is possible.

\section{5.- Experimental results}

The experimental measurements were performed under conditions corresponding to those used in the numerical simulations. The results are shown in Fig. 4, which are directly comparable with the theoretical ones in Fig. 3. The field angular distributions for varying frequency were experimentally recorded, showing agreement with the corresponding numerical simulations results shown in Fig. 3. Due to the mirror symmetry of the experimental set-up, the measurements were performed only in a half hemispace (from $0^{\circ}$ to $90^{\circ}$ ), and the figure was completed with its mirror image in order to have a straightforward comparison with the results in Fig. 3.

Figures 4(b)-(d) show the angular sound field distribution at a distance $R=1 \mathrm{~m}$ from the output face of the SC at the particular frequencies $f=5158,5543$ and $6043 \mathrm{~Hz}$ (solid lines), and illustrate the spatial filtering effects in propagation through the SC. The spatially filtered angular regions are shaded. In all cases, the transmission through the crystal is compared with the free propagation (shown by dashed line). Note that for instance, the beam shown in Figure 4 (b) presents a $-30 \mathrm{~dB}$ minimum at $30^{\circ}$. This filtering angle defines the separation between the central beam and side lobes.

\section{6.- Conclusion}

We report the first experimental demonstration of spatial filtering of sound beams in acoustics, using the SCs as a low-angle-pass spatial filter. We support the experimental measurements by numerical simulations.

The result could be of technological utility in applied acoustics, since beams of good spatial quality are desired for numerous applications. High quality beams are of lower propagation divergence, and can be focused into a tighter spot. They present also 
reduced side-lobes (or no side-lobes in an ideal case). All these properties improve the performance of ultrasonic applications (medical, as surgery or imaging, or nondestructive testing). We demonstrate, as a prototype, the formation of a beam of a good spatial quality, which is obtained from a source with a really "bad" angular distribution of the emission.

The formation of a good spatial quality beam is possible in a narrow frequency range for a particular SC (with fixed parameters such as filling factor and geometry). Then particular angular areas are filtered out from the angular spectrum of the source. If the source has a too broad angular spectrum (significantly broader than the angular bandgaps) then multiple filtering is possible by a serial propagation through two or several SC filters. Also chirped SCs could be used, where the longitudinal period or/and filling factor varies continuously along the SC. The study of filtering by chirped SCs is in progress.

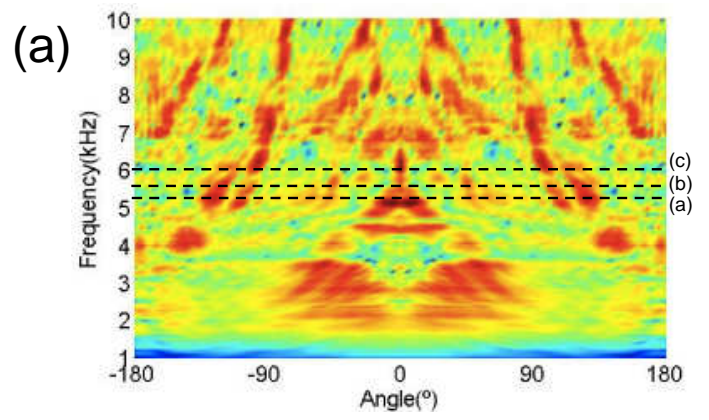

(b)

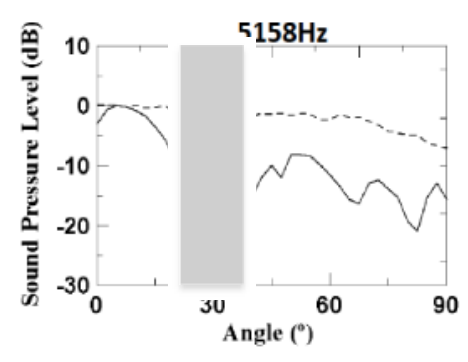

(c)

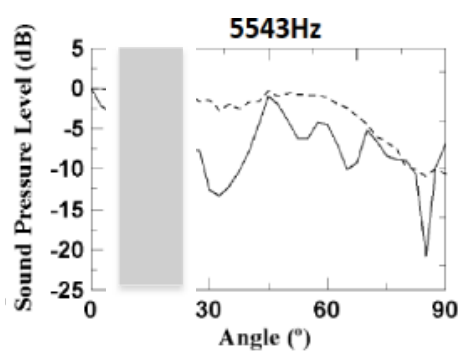

(d)

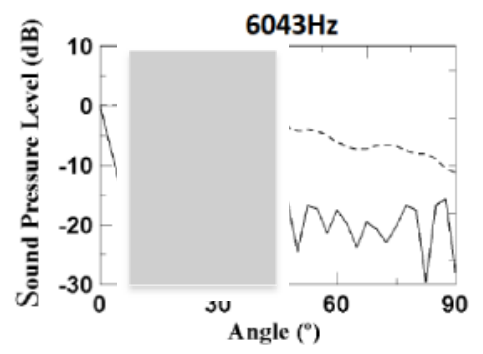


Fig.4. Experimental results evidencing the spatial filtering. In (a) the angle-frequency scan is shown, as in Fig. 3. Figs. (b)-(d) show the sound pressure level, with (continuous line) and without crystal (dashed line) at the distance $R=1 \mathrm{~m}$ from the end of the sonic crystal (as illustrated in Fig. 1).

The phenomenon has been demonstrated for two-dimensional SCs. However the band calculation of the three-dimensional SCs shows the existence of the twodimensional angular band gaps, as well as full two-dimensional self-collimation of the beams [14]. This envisages the possibility of the full two-dimensional spatial filtering with three-dimensional SCs, which could be highly utile in technological applications. The corresponding experiments are in advance.

\section{Acknlowledgements}

The work was financially supported by Spanish Ministerio de Ciencia e Innovación and by European Union FEDER through projects FIS2008-06024-C02-02 and -03. Support in experimental part of this work from Q. Lamome and M. Contraire is acknowledged.

\section{References:}

[1] Y.J. Lee, J. Yeo, R. Mittra, and W.S. Park, IEEE Trans. Antennas Propag. 53, 224 (2005).

[2] K. Staliunas and V.J. Sánchez-Morcillo, Phys. Rev. A 79, 053807 (2009);

[3] A.E. Serebryannikov, A.Y. Petrov, and E. Ozbay, Appl. Phys. Lett. 94, 181101 (2009).

[4] E. Colak, A.O. Cakmak, A.E. Serebryannikov, and E. Ozbay, J. Appl. Phys. 108, 113106 (2010)

[5] E.Yablonovitch, Phys. Rev. Lett. 58, 2059 (1987); S. John, Phys. Rev. Lett. 58, 2486 (1987);

[6] J.V. Sánchez-Pérez, D. Caballero, R. Martínez-Sánchez, C. Rubio, J. SánchezDehesa, F. Meseguer, J. Llinares and F. Gálvez. Phys. Rev. Lett. 80, 5325 (1998)

[7] L. Maigyte, T. Gertus, M. Peckus, J. Trull, C. Cojocaru, V. Sirutkaitis, and K. Staliunas, Phys. Rev. A 82, 043819 (2010)

[8] K. Staliunas, Phys.Rev.A 84, 013626 (2011)

[9] R. Picó, V.J. Sánchez-Morcillo, I. Pérez-Arjona and K. Staliunas, Applied Acoustics 73, 302 (2012);

[10] M.S. Kushwaha, P. Halevi, Appl. Phys. Lett. 69, 31 (1996) 
[11] I. Pérez-Arjona, V.J. Sánchez-Morcillo, J. Redondo, V. Espinosa and K. Staliunas, Phys. Rev. B 75, 014304 (2007)

[12] V. Espinosa, V.J. Sánchez-Morcillo, K. Staliunas, I. Pérez-Arjona and J. Redondo, Phys. Rev. B 76, 140302(R) (2007)

[13] The commercial package COMSOL Multiphysics 3.3a was used.

[14] E. Soliveres, V. Espinosa, I. Pérez-Arjona,V.J. Sánchez-Morcillo and K. Staliunas, Appl. Phys. Lett. 94, 164101 (2009) 